\title{
Hybrid Wing Body Planform Design with Vehicle Sketch Pad
}

\author{
Douglas P. Wells ${ }^{1}$ \\ NASA Langley Research Center, Hampton, Virginia 23681 \\ Erik D. Olson ${ }^{2}$ \\ NASA Langley Research Center, Hampton, Virginia 23681
}

\begin{abstract}
The objective of this paper was to provide an update on NASA's current tools for design and analysis of hybrid wing body (HWB) aircraft with an emphasis on Vehicle Sketch Pad (VSP). NASA started HWB analysis using the Flight Optimization System (FLOPS). That capability is enhanced using Phoenix Integration's ModelCenter ${ }^{\circledR}$. Model Center enables multifidelity analysis tools to be linked as an integrated structure. Two major components are linked to FLOPS as an example; a planform discretization tool and VSP. The planform discretization tool ensures the planform is smooth and continuous. VSP is used to display the output geometry. This example shows that a smooth \& continuous HWB planform can be displayed as a three-dimensional model and rapidly sized and analyzed.
\end{abstract}

\section{Nomenclature}

$\begin{array}{ll}B W B & =\text { Blended Wing Body } \\ F L O P S & =\text { Flight Optimization System } \\ H W B & =\text { Hybrid Wing Body } \\ N A S A & =\text { National Aeronautics and Space Administration } \\ V S P & =\text { Vehicle Sketch Pad } \\ 3 D & =\text { three-dimensional } \\ b & =\text { wing span } \\ x & =\text { longitudinal (chordwise) coordinate } \\ x_{0} & =\text { baseline longitudinal (chordwise) coordinate } \\ \Delta x & =\text { change in the longitudinal (chordwise) coordinate } \\ y & =\text { lateral (spanwise) coordinate }\end{array}$

\section{Introduction}

Hybrid Wing Body (HWB) or more commonly, Blended Wing Body (BWB) concepts have been studied for over 20 years both nationally and internationally. The configuration lends itself to multiple optimization points. It has potential for noise benefits ${ }^{1}$ and aerodynamic benefits which can result in fuel savings ${ }^{2}$.

A significant amount of effort has been put into the design and exploration of this unique configuration. A large portion of this effort was captured as part of a NASA technical memorandum ${ }^{3}$ containing 60 references to systemlevel studies. Significant progress has been made since the memorandum was published. One of the major accomplishments was the Boeing Phantom Works/NASA/Air Force Research Laboratory wind tunnel and flight tests of the X-48B. While scale models have been built and tested, no full scale aircraft exist to date.

\footnotetext{
${ }^{1}$ Aerospace Engineer, Aeronautics Systems Analysis Branch, MS 442, AIAA Member.

${ }^{2}$ Senior Research Engineer, Aeronautics Systems Analysis Branch, MS 442, AIAA Senior Member.
} 
To support the continuing development of HWB configuration aircraft, there is a need to develop better analysis tools and methods that address the current uncertainties. Uncertainty can be reduced through the application of test data to design and analysis tools and methods. NASA aeronautics currently uses the Flight Optimization System

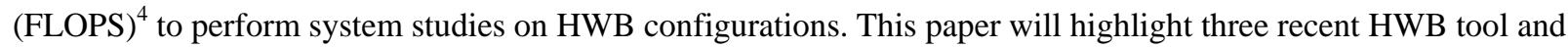
method developments that enhance HWB design, a planform control technique, geometry modeling software, and an analysis integration structure.

\section{HWB Planform Parameterization for FLOPS}

NASA's Flight Optimization System (FLOPS) was modified to allow rapid sizing and analysis of HWB configuration aircraft. The modifications include modeling a noncircular pressurized centerbody, the outboard wing sections, and weight and drag estimation routines. The modifications are detailed in Ref. 2 . It should be noted that the new capabilities were used to compare results with a Boeing proprietary report and some differences were found, mainly in the drag estimation.

\section{HWB Planform Discretization}

An efficient HWB planform accomplishes a smooth transition from the centerbody shape enclosing the payload, to the trapezoidal outer wing. For maximum aerodynamic efficiency, the planform shape particularly the leading edge should exhibit continuous sweep and curvature across the span. However, in order to perform an optimization of the planform, the number of design variables needed to describe these smooth curves can quickly become unwieldy. Instead of specifying the parameters at a large number of cross-sections, a method has been devised to discretize an HWB planform based on a smaller number of variables but still maintain its smooth shape. The leading and trailing edges of the planform are described by a set of control points, which are linked by clamped cubic splines of the form $x=x(y)$. The cubic splines exhibit positional, slope and curvature continuity across the span and thus the discretization does not comprise the aerodynamic efficiency of the planform. Each control point is represented by spanwise and chordwise coordinates, normalized by the semispan, and the root and tip sweep angles complete the definition of the curves. Since a typical HWB planform uses a trapezoidal outboard wing section, the outboard wing leading and trailing edges can be defined by straight lines, thus forcing the second- and third-order terms of the outboard splines to be zero.

Figure 1 shows a sample HWB planform defined by

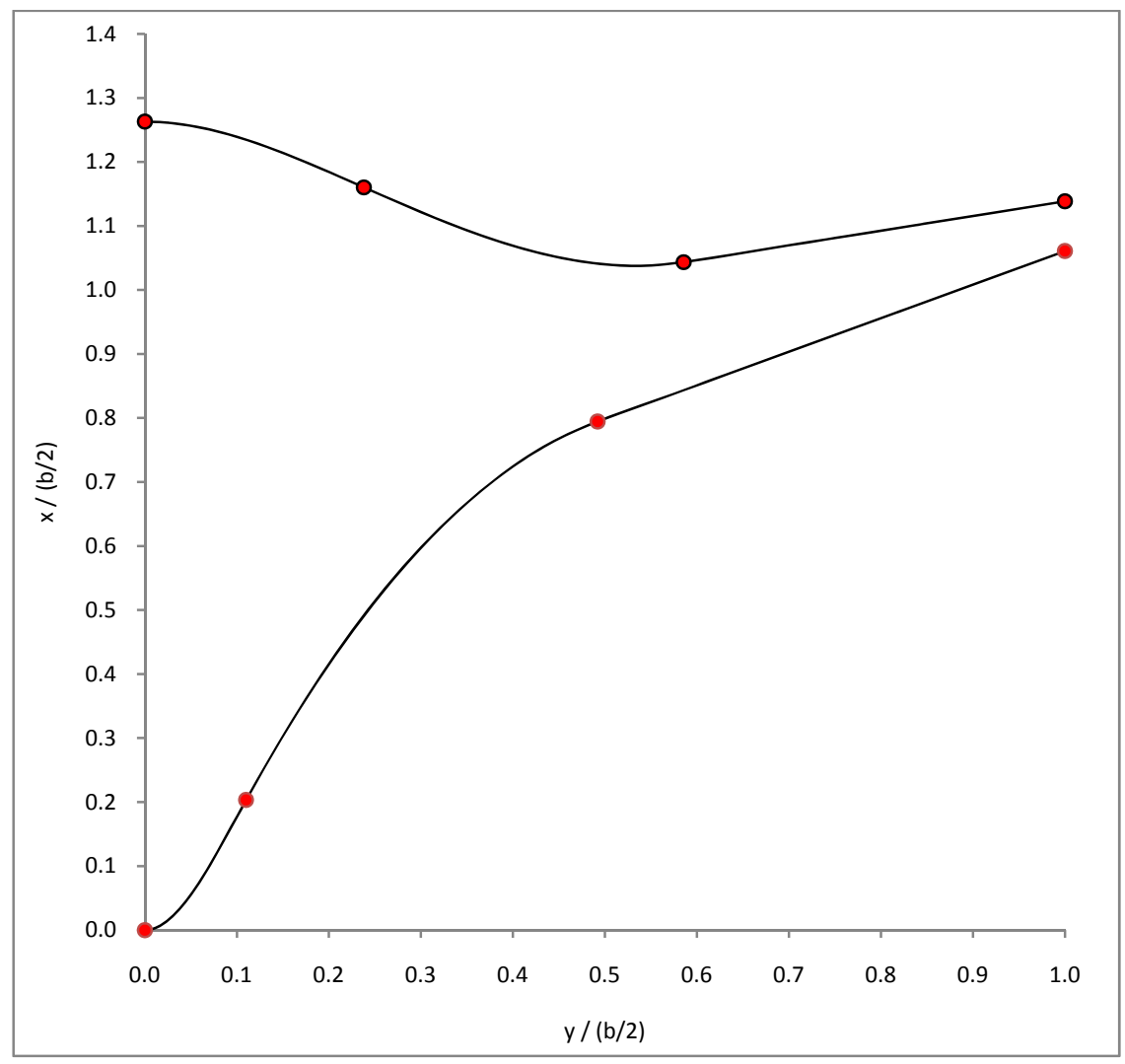

Figure 1. HWB planform discretization using direct spline fits. 
four control points each for the leading and trailing edges; with a $y$ - and an x-coordinate for each control point, resulting in 24 independent quantities to be defined. However, if the root chord is prescribed, the centerline control points are fixed except for the trailing edge centerline sweep, which typically will be zero. Also, since the span is prescribed and the outboard wing section is trapezoidal, the leading and trailing edge locations of the wing tip are entirely defined by the other design variables. Thus the full two-dimensional, smooth shape of the planform can be defined with a total of 15 design variables. Similar curves also could be defined in a similar manner to independently control the twist, camber and thickness distributions to exert more control over the full threedimensional shape of the wing during optimization.

One disadvantage to the use of splines to directly define the leading and trailing edges is that it is not possible to exactly match an existing planform of interest without an inordinate number of control points. In cases where a complex starting geometry already exists, the splines can be used instead to define transformations to the baseline planform shape:

$$
x(y)=x_{0}(y)+\Delta x(y)
$$

where $x_{0}$ is the baseline planform shape and $\Delta x$ is a transformation function defined by a cubic spline fit to a discrete set of control points. In this manner, the untransformed planform can be used to match any existing design. If the splines are set to a constant $\Delta x(y)=0$ then the planform will exactly match the baseline shape, the splines can then be used to transform the planform shape and the combined leading and trailing edges will still maintain sweep and curvature continuity.

Figure 2 shows an example of the modified discretization process. The control points for the leading- and trailing-edge splines in Figs. 2(a) and 2(b) have been kept at the same spanwise locations as before, but in this case the functions represent transformations to a baseline planform. Also, as before, the sections of the splines in the outboard wing have been constrained as first-degree polynomials to maintain the straight edges of the trapezoidal wing section. Figure 2(c) compares the baseline and transformed planform shapes, demonstrating that a more complex geometry can be defined using this modified process, while still maintaining a smooth transformed shape and limiting the number of independent variables required to fully define the full planform shape.

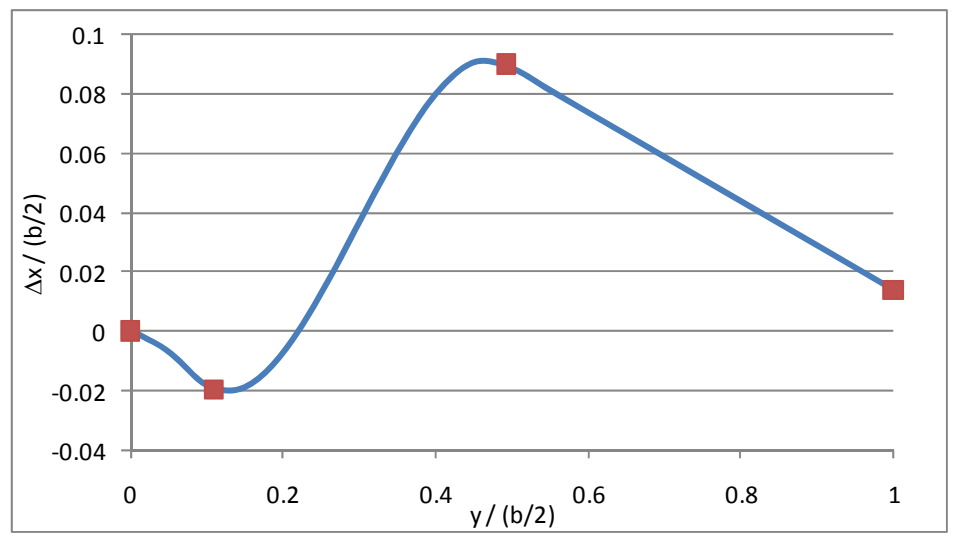

(a) Leading-edge transformation spline 


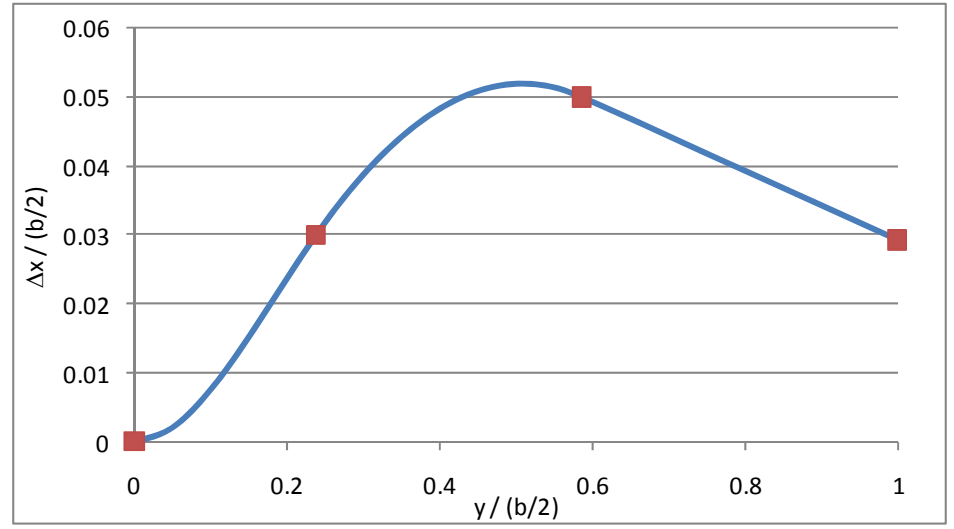

(b) Trailing-edge transformation spline

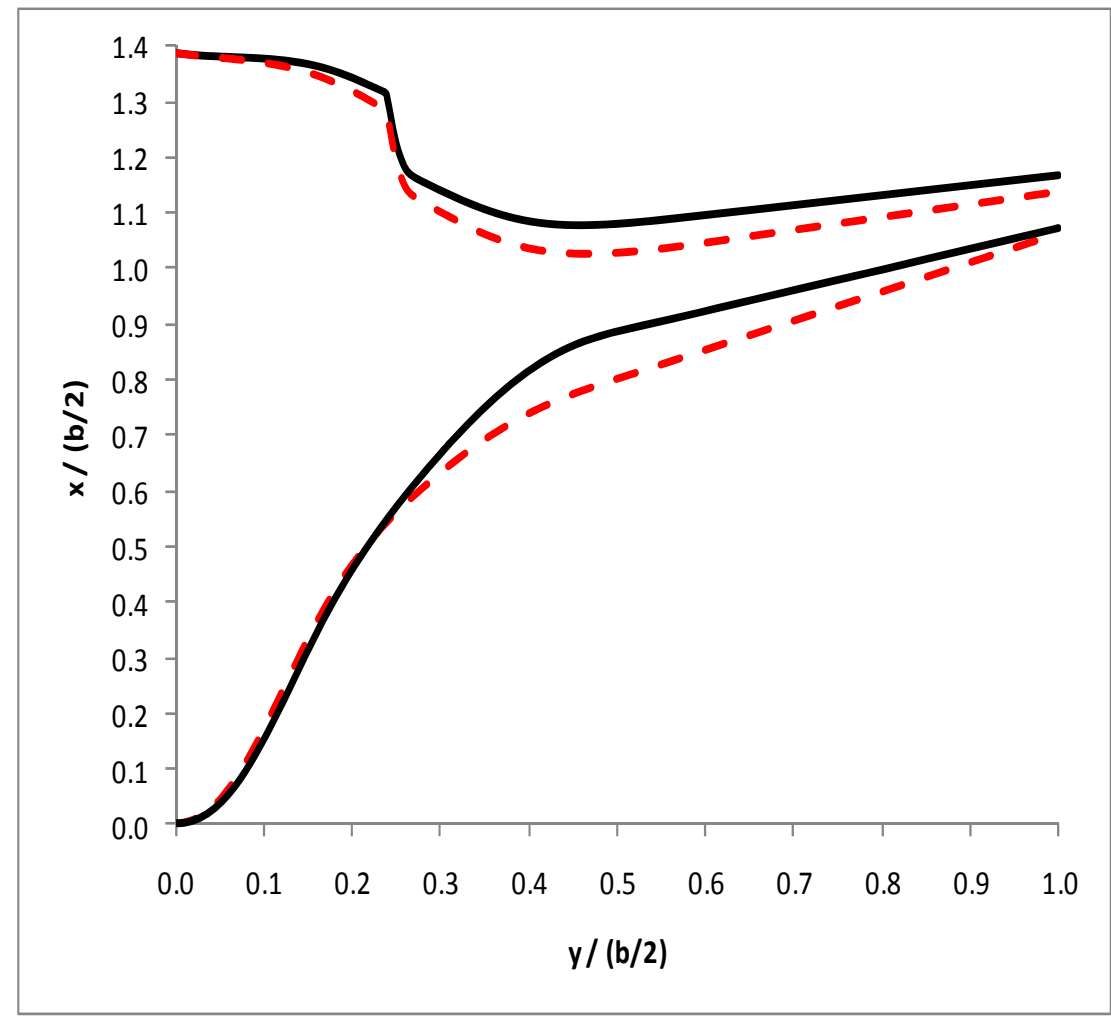

c) Original (dotted line) and modified (solid line) planform shapes

Figure 2. HWB Planform Discretization using transformation splines.

The discretization scheme is implemented as a spreadsheet method which fits the splines and outputs the span, root chord and tip chord of each of the defining aircraft geometry sections. Whenever a control point is moved, the new sectional parameters are automatically updated as output to produce a new smooth, three-dimensional, watertight representation of the configuration suitable for use in various analysis methods including computational fluid dynamics and finite element structural analysis. If used in an optimization study, constraints would be imposed to ensure that the payload can be contained within the new planform shape and that the leading and trailing edges do not cross each other and are free of inflection points. 
Figure 3 contains examples of HWB planforms that were modified using the HWB planform discretization tool. A geometry modeling software, Vehicle Sketch Pad (VSP) was linked to the discretization tool to display the output as a three-dimensional (3D) model.

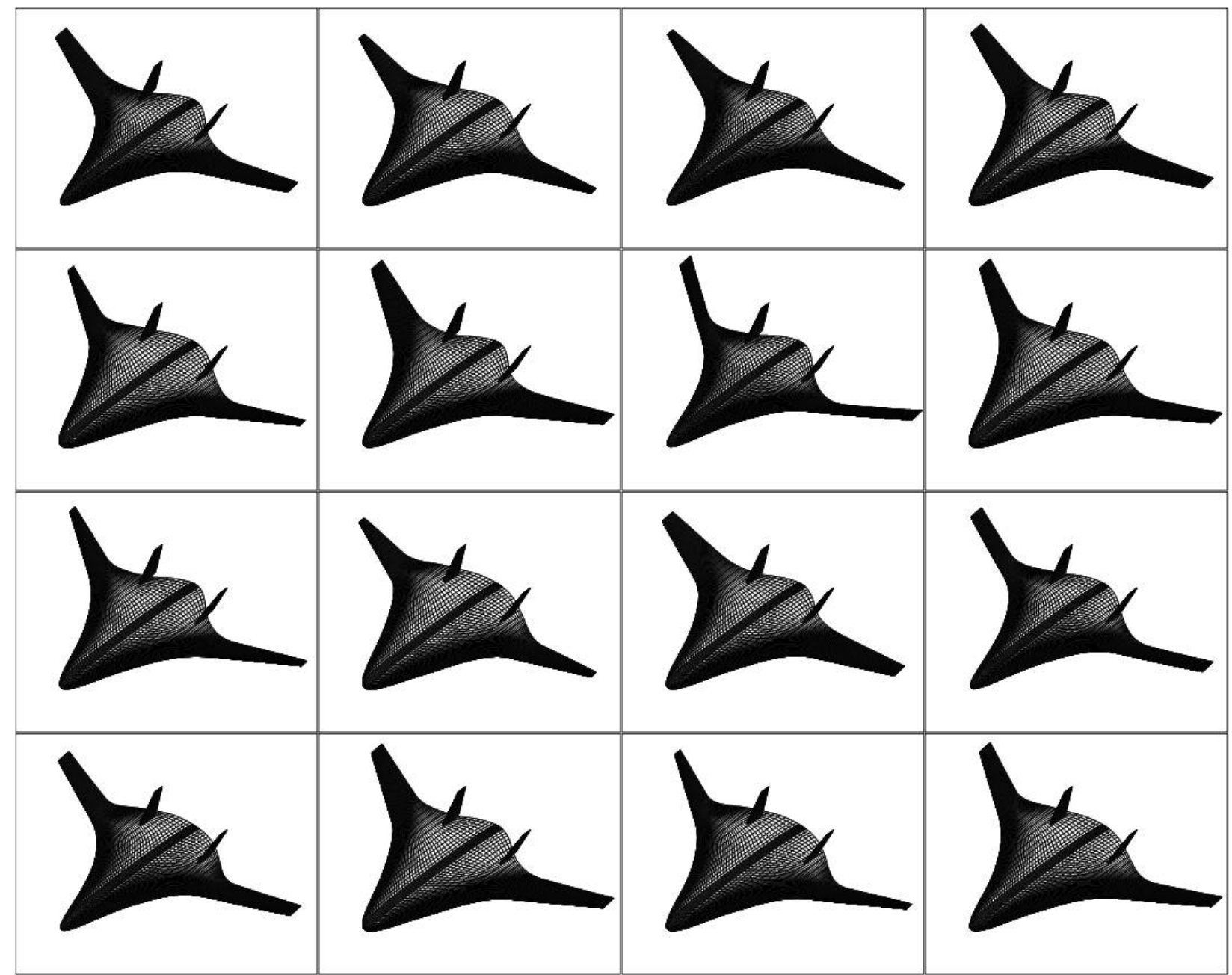

Figure 3. Sample Perturbed Planforms using HWB discretization output in VSP.

\section{Vehicle Sketch Pad}

Another development at NASA is a geometry modeling tool, VSP ${ }^{5}$. VSP is software that is used to create a parametric 3D conceptual design model. VSP was designed to be intuitive to aircraft designers with a fast and easy learning curve. Geometry models can easily be exported from VSP for use in CAD software and other higher order methods. The versatility of VSP makes it an ideal solution for most conceptual aircraft design environments.

VSP was developed by NASA Langley’s Aeronautics Systems Analysis Branch to aid in the conceptual design of the HWB configuration. Air Vehicle Integrated Design (AVID) LLC under contract developed the HWB component to address the unique geometry challenges of the HWB configuration. The HWB component is similar to the Multi Section Wing component. The dihedral tab was removed and dihedral control was moved to the section tab. Display boxes were added to the section tab for Filleted Tip Chord and Filleted Root Chord. A fillet tab was added and is shown in Fig. 4. On this tab the joint location to be modified is selected in the Joint ID box. Sweep Fillets, Trailing Edge Sweep Fillets, and Dihedral Fillets are controlled by a slider bar that sets the size of the fillet from percent wing span and an input box to set the fineness from the degrees per segment text box. There is also an 
on/off option to rotate the airfoils in the fillet to match the dihedral for interpolation control. The Sweep Fillets controls will create a fillet for the leading edge by adding section interpolations and changing the chord length at the joint. When using the Sweep Fillets, the trailing edge fillet and location are both fixed. When using the Trailing Edge Sweep Fillets, the leading edge fillet and location are both fixed. The Trailing Edge Sweep Fillets controls will create a fillet at the trailing edge in the same manner as the Sweep Fillets. The Dihedral Fillets controls will add interpolation sections and move the airfoils in the z-direction to blend the dihedral.

Figure 5 shows each of the fillets on the HWB component. Smoothing and blending is important for an HWB configuration because the performance is highly dependent on the shape and surface of the configuration. The HWB component enabled the capability to smooth the planform and dihedral angles, creating more efficient and clean geometries.

Phoenix Integration created a plug-in for VSP for use in their ModelCenter ${ }^{\circledR}$ software. The plug-in enables VSP to be integrated early in the design process. The VSP Plug-in can be obtained from Phoenix Integration with the purchase of ModelCenter ${ }^{\circledR}$ 9.0.

\section{Integration}

NASA had three tools ready for design and analysis with HWB concepts. FLOPS can size and analyze the concept, VSP can build a model of the concept, and the discretization can ensure a smooth and

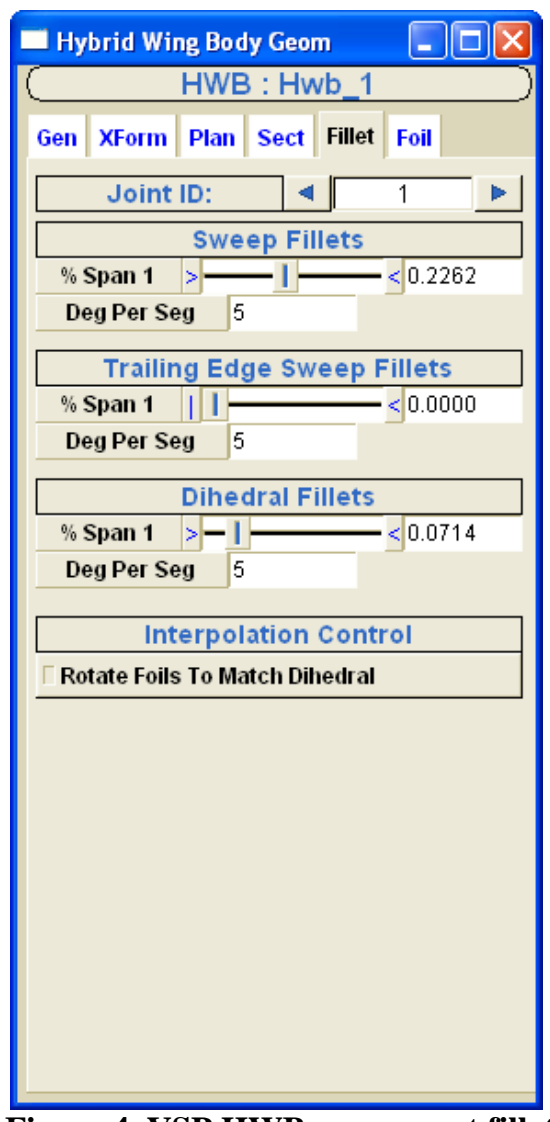

Figure 4. VSP HWB component fillet tab.

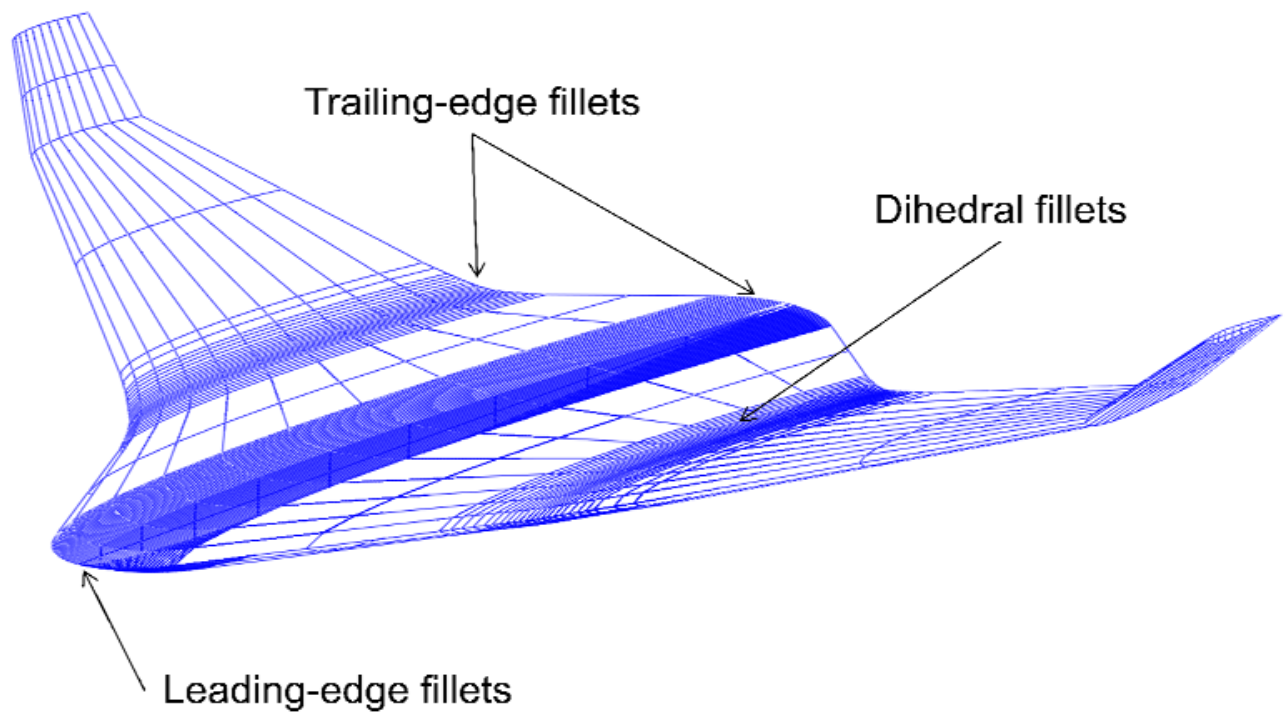

Figure 5. AVID VSP HWB Component. 
continuous planform. Once they were at a robust level, the new design and analysis tools were ready for the next step: integration.

Phoenix Integration's ModelCenter ${ }^{\circledR}$ is used as an integration environment for Multidisciplinary Analysis and Optimization (MA\&O). ModelCenter ${ }^{\circledR}$ can link multiple analysis software tools together to create an integrated design process. NASA uses ModelCenter ${ }^{\circledR}$ to integrate aircraft design tools like FLOPS, VSP, PMARC ${ }^{6}$ (aerodynamic panel program), VorView ${ }^{7}$ (adaptation of VorLax, an aerodynamic panel program), NPSS ${ }^{8}$ (engine analysis program), and others.

Figure 6 shows a sample ModelCenter ${ }^{\circledR}$ model for an HWB design process. In this sample, design variables are input, which are fed to the Geometry Parameters spreadsheet. The spreadsheet uses default data to create a smooth and continuous planform. That data is sent to VSP and a model is shown in the ModelCenter ${ }^{\circledR}$ model for visualization. Next, the data goes to the Detailed Wing component where the wing data is translated to FLOPS input. A default FLOPS input file is then read at the input file component. The geometry data from the previous components is now overwritten in the FLOPS input file at the flops component. The FLOPS analysis is run and the output is compared to the FLOPS default output.

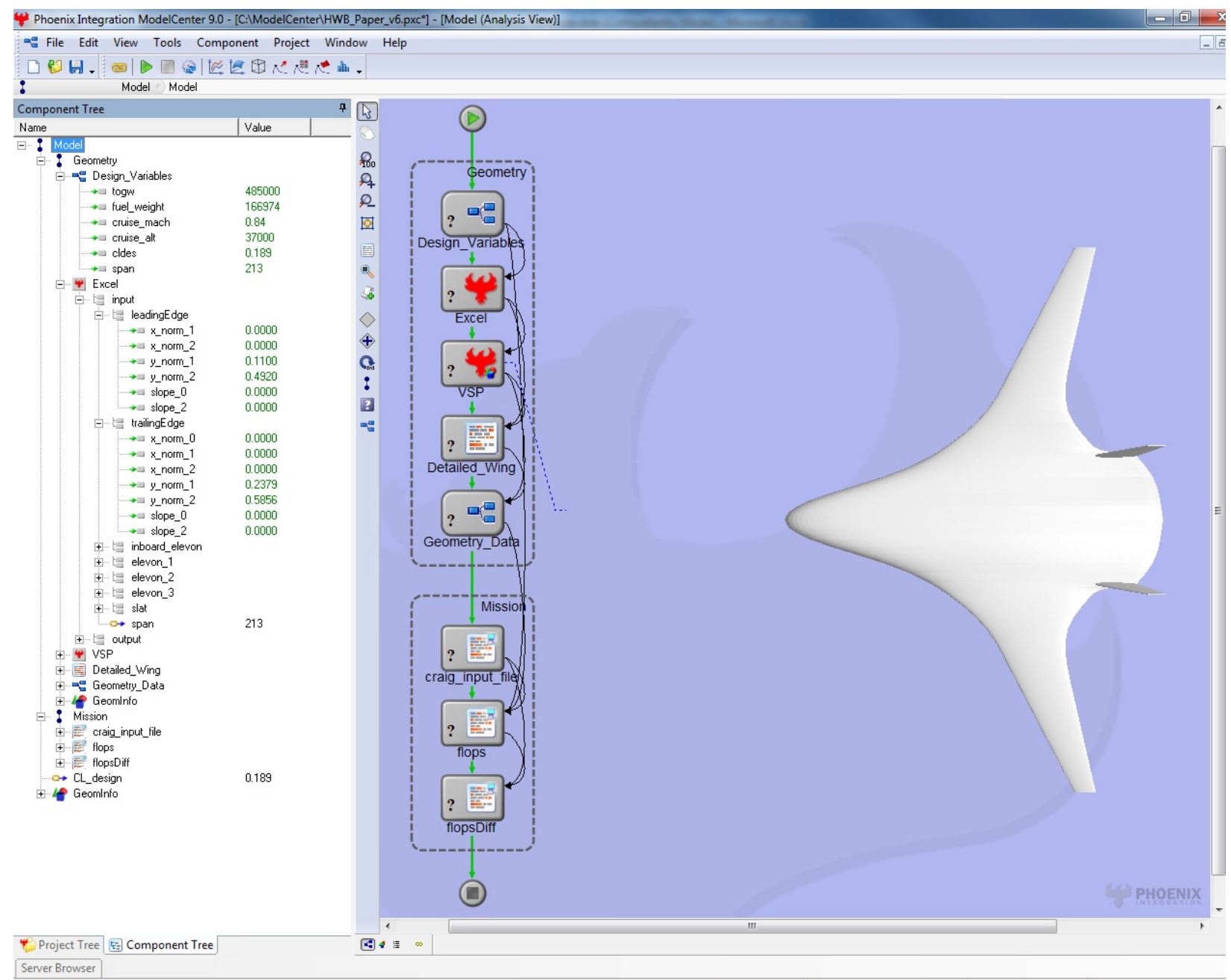

Figure 6. A sample HWB design process in ModelCenter ${ }^{\circledR}$.

Additional disciplines should be integrated into the design process the reduce risk in the design. This effort is already underway through the GEN2 toolset. The GEN2 toolset is the integration of several low, intermediate, and 
high order modules. In the GEN2 toolset, engine data is created and then the cabin is laid out. That information is used to determine the planform of the HWB using the Geometry Parameters spreadsheet. Next the data is passed to VSP where a 3D geometry model is created. The next step is to output the geometry data from VSP to FLOPS and VorView where flight performance is determined. The data is also used for structural, mission, and noise analysis. Several higher order codes may also be added such as FUN3D ${ }^{9}$ and CART3D ${ }^{10}$ (3D flow solvers).

\section{Conclusion}

NASA plans to integrate all current tools and methods using ModelCenter ${ }^{\circledR}$ into an MA\&O HWB configuration design process. The GEN2 toolset, once fully integrated will address that need. Experimental data will be used to build confidence in the toolset.

Phoenix Integration's ModelCenter ${ }^{\circledR}$ allows an integrated environment for HWB MA\&O. The capability to create an HWB planform using 15 parameters enables more efficient and rapid conceptual design. VSP's HWB component enables the creation of accurate and efficient HWB 3D models. The new HWB design capabilities presented in this paper will allow engineers to make quick changes to a conceptual HWB design and get accurate, integrated results.

NASA is working on studies that will further enhance HWB analysis capabilities. Data from the studies will be used to calibrate the analysis tools. Current efforts include open rotor engine design and structural and weight analysis and optimization. These enhancements will enable NASA to study HWB concepts with less uncertainty.

\section{Acknowledgments}

The authors would like to thank the following engineers for their valuable contributions to this capability: Craig Nickol (NASA Langley Research Center), Linwood A. McCullers (Alliant Techsystems Inc.), and JR Gloudemans. In addition, the authors would also like to thank Phoenix Integration and AVID for working with NASA to enhance analysis capabilities.

\section{References}

${ }^{1}$ Thomas, R. H., Burley, C. L., and Olson, E. D., "Hybrid Wing Body Aircraft System Noise Assessment With Propulsion Airframe Aeroacoustic Experiments,” AIAA Paper No. 2010-3913, Jun. 2010.

${ }^{2}$ Nickol, C. L. and McCullers, L. A., “Hybrid Wing Body Configuration System Studies,” AIAA Paper No. 2009-931, Jan. 2009.

${ }^{3}$ Nickol, C. L., “Silent Aircraft Initiative Concept Risk Assessment,” NASA TM-2008-215112, Feb. 2008.

${ }^{4}$ McCullers, L. A., “FLOPS Flight Optimization System, FLOPS manual.doc,” FLOPS User Manual, updated Dec. 2009.

${ }^{5}$ Hahn, A. S., “Vehicle Sketch Pad: A Parametric Geometry Modeler for Conceptual Aircraft Design,” AIAA Paper No. 2010-657, Jan. 2010.

${ }^{6}$ Ashby, D., Dudley, M., Iguchi, S., Browne, L., and Katz, J., "Potential Flow Theory and Operation Guide for the Panel Code PMARCJ2," NASA TM-102851, Dec. 1992

${ }^{7}$ Feagin, R. C., “Delta Method, An Empirical Drag Buildup Technique,” NASA CR-151971, 1978.

${ }^{8}$ Evans, A. L., Pollen, G., Naiman, C., and Lopez, I., “Numerical Propulsion System Simulation's National Cycle Program,” AIAA Paper No. 98-3113, 1998.

9 “FUN3D Manual,” updated Dec. 2010.

${ }^{10}$ Aftosmis, M. J., Berger, M. J., and Adomavicius, G., “A Parallel Multilevel Method for Adaptively Refined Cartesian Grids with Embedded Boundaries,” AIAA Paper No. 2000-0808, Jan. 2000. 